\title{
INFLUENCE OF AGE AND MORPHOLOGICAL FEATURES ON THE CLINICAL MANIFESTATIONS AND TREATMENT EFFICACY OF HEMANGIOMAS IN CHILDREN
}

\author{
Vivcharuk V., Davydenko V. \\ Kharkiv National Medical University \\ https://doi.org/10.35339/ic.8.1.22-29
}

\begin{abstract}
Background. Infantile hemangioma (IH) is a neoplasm that is most common in childhood. Morphologically, hemangiomas are divided into superficial, deep and mixed, focal, segmental, indeterminate and multifocal. The course of IH includes phases of rapid growth followed by slow involution. The degree of involution of hemangioma is variable. Hemangiomas can lead to the appearance of permanent deformities of soft tissues or functional disorders, especially when localized on the face and vital structures. Clinical heterogeneity of hemangiomas creates significant difficulties for physicians in resolving issues of treatment tactics. Specific characteristics of the clinical behavior of hemangiomas of various morphological types can be crucial in the choice of management tactics for such patients. Objectives. The aim of the work is to study the effect of different morphological types of hemangioma in children on the effectiveness of treatment of age-related clinical features. Subjects and Methods. The study group consisted of 100 children in age from birth to 6 years who have hemangiomas of different morphological types and localizations and did not receive previous treatment. All research participants for hemangioma severity scale (HSS), hemangioma activity scale (HAS), visual analogue scale (VAS) before, during and after treatment were scored. Serum levels $\mathrm{sFas} / \mathrm{sFasLs}$ before and during treatment evaluated. Results. With increasing the child's age, the indicator as for HAS reliably decreases for all types of hemangiomas. In all types of hemangiomas predominantly an active growth took place. An abortive growth in $10 \%$ of patients was noted. For focal hemangiomas, the indicator of sFas decreases with age, and for multifocal hemangiomas it increases. In both cases, there is an increase in the sFasL indicator with increasing age of the child. In our study, in age group before 1 year in the factor structures, "age" factor was considered to be the main one and manifested by a decrease in the activity of hemangioma with an increase in the age of the child. Among children over 1 year age, during the course of treatment, the greatest contribution the "hemangioma severity factor", the influence of which leads to a decrease in the cosmetic effect of hemangioma treatment against the background of its high severity and an increase in the indicator of apoptosis inhibition of sFas. The influence of the "treatment efficacy" factor leads to a decrease in its cosmetic effect and an increase in the severity of the manifestations of hemangioma against the background of blocking the manifestations of apoptosis. Conclusions. When starting treatment of hemangioma in the early stages, the cosmetic effect increases significantly. A decrease in sFasL in the younger age group may indicate a decrease or absence of apoptosis processes, which is clinically expressed by active proliferation. An increase in sFas in the older age group (over a year old) may indicate a blockage of apoptosis processes and, as a consequence, a slow regression of hemangioma. Keywords: hemangioma, children, morphological type, age, sFas/sFasL, hemangioma activity scale, visual analogue scale.
\end{abstract}

Corresponding Author:

Viktoriia Vivcharuk, MD, PhD student

of the Department of Pediatric Surgery

and Pediatric anesthesiology, Kharkiv National

Medical University, Kharkiv, Ukraine.

E-mail: viktoriia.doc@gmail.com 


\section{Introduction}

Infantile hemangioma $(\mathrm{IH})$ is a neoplasm that is most common in childhood [1,2]. According to the 2018 classification of the International Society for the Study of Vascular Anomalies (ISSVA), hemangiomas are considered to be benign vascular tumors. The latter include infantile hemangiomas and congenital hemangiomas of three types: rapidly involuting (RICH), partially involuting (PICH), non-involuting (NICH) [3].

Hemangiomas in clinical practice differ in localization, morphological type and stage of development [4]. Morphologically, hemangiomas are divided into superficial, deep and mixed, focal, segmental, indeterminate and multifocal $[5,6]$. The predominant localization of hemangiomas is on the head, face, neck $[7,8]$.

The characteristic course of IH includes phases of rapid growth due to proliferation followed by slow involution. Most hemangiomas have the first clinical manifestations in the form of pale spots, telangiectasias, pink macules, "scratches" or "bruises" in the neonatal period, followed by the rapid development of the tumor $[9,10]$.

In general, hemangiomas proliferate by the age of 6-9 months [11]. According to some studies, the growth of hemangiomas ends at the age of 3 months [12], according to others, the completion of their growth was observed at 5 months [13]. Subcutaneous hemangiomas, in comparison with superficial ones, have a much longer proliferative activity [14], sometimes up to 14 months and even over 2 years. The growth of hemangiomas in children over 3 years of age is an atypical phenomenon [15].

The degree of involution of hemangioma is variable. Recent studies have shown that in untreated hemangioma, in almost $90 \%$ of cases, involution is completed by 4 years of age [16].

Despite the fact that most hemangiomas do not cause considerable problems, a significant part of them can lead to the appearance of permanent deformities of soft tissues or functional disorders, especially when localized on the face and vital structures $[17,18]$. The presence or consequences of hemangiomas can affect the quality of life of both children and their parents due to anxiety, low self-esteem of children and peer bullying due cosmetic defects [19].

The average age of contacting a pediatric dermatologist is 5 months, at this time the main growth and possible complications are already developing $[20,21]$. Clinical heterogeneity of hemangiomas creates significant difficulties for physicians in resolving issues of treatment tactics [22, 23].
For hemangiomas that require treatment, the best time to start is before or immediately after the onset of obvious risks of complications [24]. It was found that IH treatment in the proliferative phase allows achieving the best result $[25,26]$.

Despite the fact that the general signs of the course of hemangiomas have been studied for a long time, there is not enough description of the specific details of the growth of hemangiomas and information about the variety of manifestations of growth among hemangiomas of different subtypes. Specific characteristics of the clinical behavior of hemangiomas of various morphological types can be crucial in the choice of management tactics for such patients.

2. Purpose, subjects and methods:

2.1. The purpose of the work is to study the effect of different morphological types of hemangioma in children on the effectiveness of treatment of age-related clinical features.

\subsection{Subjects \& Methods}

The study group consisted of 100 children who were consulted on an outpatient basis and who received treatment at the Regional Children Clinical Hospital No. 1 in Kharkiv from September 2017 to October 2019. The selection criteria were: the age of children from birth to 6 years, children who had neoplasms with clinical signs of hemangiomas of external location, their complications and consequences, children who did not receive previous treatment, children whose representatives have agreed to participate in the study.

The methods used in this study were agreed and approved by the ethics committee of Kharkiv National Medical University No. 6 dated 04.10.2017, in accordance with the 1975 Declaration of Helsinki.

In almost all cases, the diagnosis was made during an outpatient visit. The age, sex, birth weight, the period of hemangioma occurrence and its morphological type, growth peculiarities (active or abortive), the maximum size of the hemangioma, its localization and depth of spread, the presence of complications, the period of appearance of hemangioma regression signs were determined in children.

The indicators of the general clinical blood analysis, clinical urine test, blood glucose levels, $\mathrm{sFas}$ and $\mathrm{sFasL}$ indicators before and during treatment, and electrocardiography data were evaluated. Children receiving systemic propranolol therapy were consulted by a cardiologist. According to the indications, echocardiography was performed, consultations of other specialists (ophthalmologist, ENT) were appointed. 
To determine the clinical state of hemangioma, we used the severity scale (HSS), which was assessed once during the initial examination, as well as the hemangioma activity scale (HAS), which was used before the appointment of treatment (HAS1) and after three months of treatment (HAS2). The achieved cosmetic result was determined using a visual analogue scale (VAS) based on photographs taken before treatment, during its treatment (VAS1) and after completion (VAS2). A comparison of photographs taken before treatment and three months after the start of treatment was made in order to assess the dynamics of the process.

Determination of sFas and sFasL indicators in blood serum was carried out by the enzymelinked immunosorbent assay using a commercial test system manufactured by "Elabscience" (ELISA, USA) on an enzyme-linked immunosorbent analyzer "Labline-90" (Austria), according to the instructions that were added to the kit.

Taking into account the different activity of hemangiomas and their size, the treatment was prescribed according to individual indications such as: active growth of the neoplasm, cosmetic defects caused by them or obvious risks of their formation, as well as the desire of the parents to get rid of the hemangioma.

Treatment of complicated, large and active hemangiomas was carried out according to the appointment of systemic therapy with propranolol (17 children). Children with superficial flat lesions were prescribed topical treatment with $0.5 \%$ timolol maleate solution ( 15 children). In cases of focal (deep, mixed and superficial), some multifocal and indeterminate hemangiomas, protruding above the skin surface and spread subcutaneously, combined treatment with $0.5 \%$ timolol maleate solution and local compression was prescribed ( 52 children). Children with volumetric formations up to $1 \mathrm{~cm}$ in diameter underwent intratumor administration of triamcinolone acetonide, followed by topical application of $0.5 \%$ timolol maleate solution ( 8 children). Intratumor administration of triamcinolone acetonide was performed in children with active mucosal hemangiomas that were small ( 1 child). Surgical removal of hemangiomas was performed in children over a year old due to the presence of a cosmetic defect and at the request of the parents ( 5 children). Two children, 3 and 5 years old, were not prescribed treatment due to unexpressed residual changes.

During the treatment, the heart rate and blood glucose levels were monitored.
Statistical processing of the research results was carried out using the Statistica 6.0 software package. Average values of indicators, standard errors, and, if necessary, medians (Me) and quartiles $(25 \%$; $75 \%)$ were calculated. Nonparametric Wilcoxon, Mann-Whitney, Pearson $\chi^{2}$ criteria were used to compare the samples due to the absence of normal distributions of the studied indicators..

\section{Results \& Discussion}

There were $32(32 \pm 4.7) \%$ boys and 68 $(68 \pm 4.7) \%$ girls in the study group. Children aged 0-6 months - $62(62 \pm 4.9) \%$ persons consisted the largest age group; there were $17(17 \pm 3.8) \%$ children aged $7-12$ months; $18(18 \pm 3.8) \%$ children aged $13-36$ months and $3(3.0 \pm 1.7) \%$ children aged 37-72 months.

The children of the study group had four morphological types of hemangiomas: focal $71(71 \pm 4.5) \%$ persons; segmental $-4(4.0 \pm 2.0) \%$ persons; indeterminate $-7(7.0 \pm 2.6) \%$ persons; multifocal $-18(18 \pm 3.8) \%$ people. Thus, children with focal and multifocal hemangiomas consisted the significant $(p<0.05)$ majority in the study group.

The average values of the indicator as for HAS1, taking into account the age of the child and the morphotype of the hemangioma are given in Table 1.

The table shows that with increasing the child's age, the indicator as for HAS1 reliably decreases for all types of hemangiomas.

In 30 children, abortive growth of hemangioma was noted, among them in 22 up to the age of 6 months inclusively, in 6 children under 1 year and in two children at the age of 12 months. Clinical signs of independent regression were observed in 17 children with active growth and in 2 children with abortive growth of hemangiomas. In 6 children, signs of regression appeared before the age of 6 months, in 7 children - in the period from 7 to 12 months, and in 6 children - from 12 months to 24 months.

According to Table 2, it can be noted that among all morphotypes of hemangioma, its active growth is reliably more often observed in both boys and girls. In the group with active growth, girls are reliably more common than boys.

The results of the study of the dynamics of Fas and FasL indicators in children of different ages during treatment are shown in Fig. 1 and Fig. 2.

According to the histograms shown in Fig. 1 and Fig. 2, it can be noted that in both cases there is a tendency to change in the sFas indicator, but in different directions. For focal hemangiomas, 
Average values of the indicator as for HAS1 in age groups of children

Table 1 with different morphotypes of hemangiomas

\begin{tabular}{|c|c|c|c|c|}
\hline \multirow{2}{*}{ Morphotype } & \multicolumn{4}{|c|}{ Values of HAS1, point } \\
\hline & $0-6$ & $7-12$ & $13-36$ & $37-72$ \\
\hline Focal $(n=71)$ & $10.5 \pm 1.2$ & $\begin{array}{l}7.7 \pm 1.6^{*} \\
U=36.5\end{array}$ & $\begin{array}{c}6.1 \pm 0.9^{*} \\
U=8.5\end{array}$ & $\begin{array}{l}3.0 \pm 1.7^{* * *} \\
U=0 ; U=0\end{array}$ \\
\hline Segmental $(n=4)$ & 11.0 & 0 & 7.0 & 0 \\
\hline Indeterminate $(n=7)$ & $12.2 \pm 1.0$ & $\begin{array}{c}5.7 \pm 1.2^{*} \\
U=0\end{array}$ & 0 & 0 \\
\hline Multifocal $(n=18)$ & $9.5 \pm 1.4$ & $\begin{array}{c}7.0^{*} \\
U=3.5\end{array}$ & $\begin{array}{c}6.0 \pm 1.7^{*} \\
U=1.5\end{array}$ & 0 \\
\hline
\end{tabular}

Notes: * - differences in the values of the indicator between the age group (0-6) months and other age groups are reliable $(\mathrm{p}<0.01)$; ** - differences in the values of the indicator between the age group $(7-12)$ months and other age groups are reliable $(\mathrm{p}<0.01)$.

Distribution of children with different morphotypes

Table 2 of hemangiomas by growth characteristics, (\%)

\begin{tabular}{|c|c|c|c|c|}
\hline \multirow{3}{*}{ Morphotype } & \multicolumn{4}{|c|}{ Growth characteristics of hemangioma } \\
\hline & \multicolumn{2}{|c|}{ Active growth } & \multicolumn{2}{|c|}{ Abortive (Minimal growth) } \\
\hline & boys & girls & boys & girls \\
\hline Focal $(n=71)$ & $\begin{array}{c}21(28 \pm 5.3)^{2} \\
x^{2}=20.6\end{array}$ & $47(66 \pm 5.6)$ & $\begin{array}{c}2(4.0 \pm 2.3)^{3} \\
x^{2}=14.99\end{array}$ & $\begin{array}{c}1(2.0 \pm 1.7)^{1} \\
x^{2}=66.79\end{array}$ \\
\hline Segmental $(n=4)$ & $1(25 \pm 21.7)$ & $1(25 \pm 21.7)$ & $1(25 \pm 21.7)$ & $1(25 \pm 21.7)$ \\
\hline Indeterminate $(n=7)$ & $\begin{array}{c}0^{2} \\
x^{2}=10.5\end{array}$ & $6(86 \pm 13)$ & 0 & $\begin{array}{c}1(14 \pm 13)^{1} \\
x^{2}=7.14\end{array}$ \\
\hline Multifocal $(n=18)$ & $\begin{array}{c}4(22 \pm 9.8)^{2} \\
X^{2}=5.6\end{array}$ & $11(61 \pm 11.5)$ & $3(17 \pm 8.9)$ & $\begin{array}{c}0^{1} \\
x^{2}=15.84\end{array}$ \\
\hline Total $(n=100)$ & $\begin{array}{c}25(25 \pm 4.3)^{2} \\
x^{2}=32.32\end{array}$ & $65(65 \pm 4.8)$ & $\begin{array}{c}7(7.0 \pm 2.6)^{3} \\
x^{2}=12.05\end{array}$ & $\begin{array}{c}3(3.0 \pm 1.7)^{1} \\
x^{2}=85.65\end{array}$ \\
\hline
\end{tabular}

Note: 1 - differences in the incidence of active and minimal hemangioma growth in girls are reliable $(\mathrm{p}<0.05) ; 2$ - differences in the incidence of active hemangioma growth between girls and boys are reliable $(\mathrm{p}<0.05) ; 3$ - differences in the incidence of active and minimal growth of hemangiomas in boys are reliable $(\mathrm{p}<0.05)$.

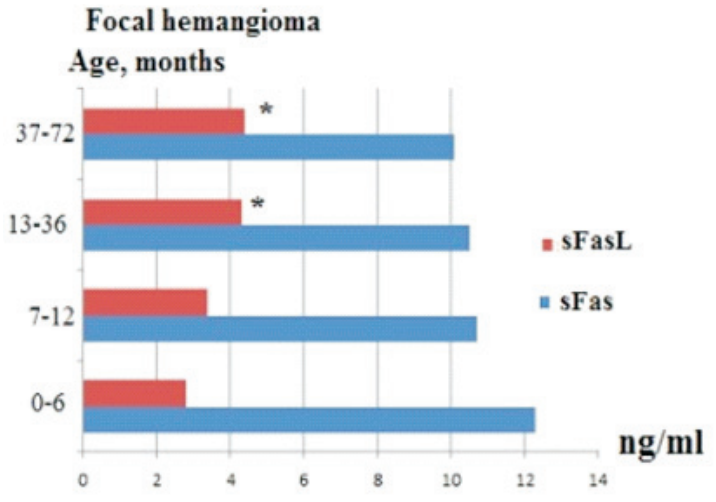

Fig. 1. Age-related dynamics of sFas and sFasL indicators in children with focal hemangioma.

* - differences in the sFasL content in the blood of children of the age group (0-6) months and children of other age groups are reliable according to the Mann-Whitney criteria $(\mathrm{p}<0.01)$

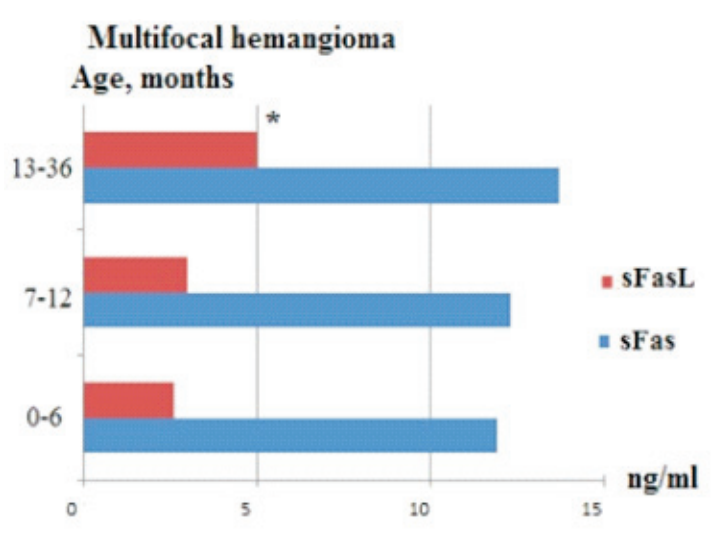

Fig. 2. Age-related dynamics of sFas and sFasL indicators in children with multifocal hemangioma. * - differences in the sFasL content in the blood of children of the age group (0-6) months and children of other age groups are reliable according to the Mann-Whitney criteria $(\mathrm{p}<0.01)$ 
the indicator decreases with age, and for multifocal hemangiomas it increases. In both cases, there is an increase in the sFasL indicator with increasing age of the child. According to this indicator, reliable differences were revealed between the first and third, and the first and fourth age groups for the focal hemangioma, and between the first and third - for the multifocal one. There were no reliable differences between the indicators for other morphotypes of hemangioma, which is associated with the insufficient volume of the respective groups of children.

To identify the relationship of clinical history and laboratory indicators in children of different ages, factor analysis was carried out and factor structures were plotted (Fig. 3). Since the distribution of the proportion of the studied indicators did not correspond to the normal one, the pairwise Spearman's correlation coefficients were used as input indicators.

Currently, studies are being done on various factors, including biomarkers that affect: the clinical behavior of hemangiomas during treatment [27-29], the development of unwanted side effects during treatment [30], the risk of complications [31], and factors of the appearance of hemangioma in general [32].

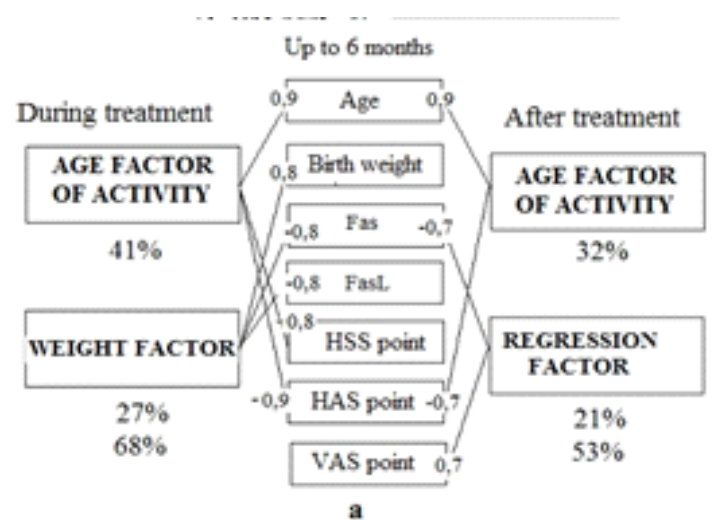

In our study, in all age groups in the factor structures, two factors were found, which indicates their plasticity. In the younger age group (Fig. 3,a), the first factor of the factor structure, plotted according to the indicators obtained during the course of treatment, called the "age factor of activity", explains $41 \%$ of the total variance. The action of this factor leads to a decrease in the activity and severity of hemangioma against the background of an increase in the child's age. The action of the second factor, called the "weight factor", which explains $27 \%$ of the total variance, leads to a decrease in apoptosis indicators against the background of an increase in the child's weight at birth. It is known that low birth weight is one of the risk factors for hemangioma, and it also determines the severity and activity [33]. The $\mathrm{sFas}$ and $\mathrm{sFasL}$ indicators characterize the level of intensity of apoptosis processes. The sFasL concentration can be proportional to the intensity of apoptosis processes [34]. The exact mechanism of interaction between membrane and soluble forms of Fas and FasL (sFas, sFasL) remains unknown [35]. It has been suggested that soluble Fas has an inhibitory effect on Fas/FasLmediated apoptosis [36]. The total contribution of factors to the total variance is $68 \%$, which

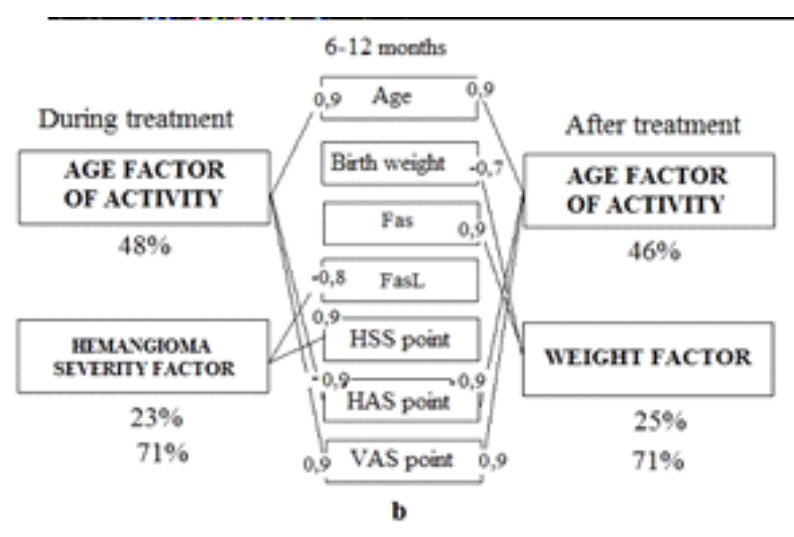

Older then 12 months

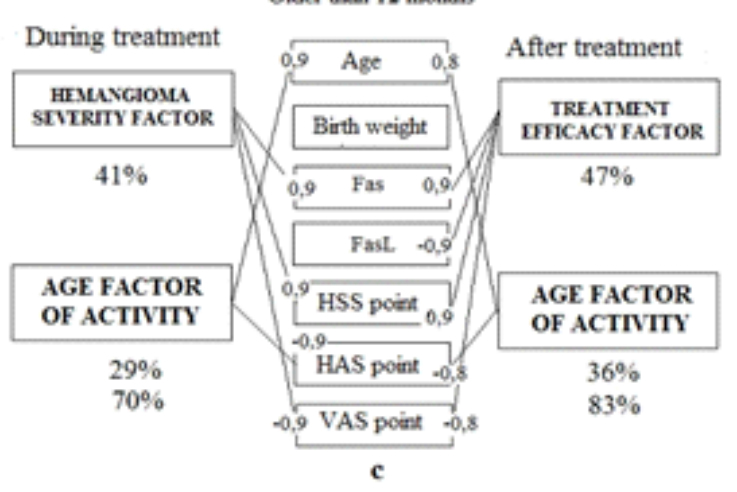

Fig. 3. Factor structures of indicators of patients of different age groups with hemangioma 
indicates the presence of a random component, which has increased after treatment. This is a sign of significant external influences on the studied system of indicators in young children. After treatment, the first factor has changed its configuration, it only affects the activity of the hemangioma, which decreases with increasing age of the child. The contribution of this factor to the total variance has decreased and is $32 \%$. The second factor, called the "regression factor", leads to an increase in the VAS indicator after treatment, which happens in parallel to a decrease in the sFas indicator.

In the second age group of children (Fig. 3,b), the action of the first factor, plotted according to the indicators obtained during the course of treatment, leads to an increase in the cosmetic effect of treatment against the background of a decrease in hemangioma activity (according to HAS) and an increase in the child's age. The contribution of this factor to the total variance is $48 \%$. The second factor of the structure - the "hemangioma severity factor" - causes an increase in the indicator according to HSS against the background of a decrease in apoptosis. The total contribution of both factors to the total variance is $71 \%$, which indicates a smaller influence of the random component on the system of the studied indicators than in the first age group (Fig. 3,a). The action of the first factor of the structure, plotted according to the indicators obtained after treatment, leads to an improvement in the results of treatment with an increase in the child's age against the background of a decrease in hemangioma activity (according to HAS). The structure of relationships in the system of indicators in the factor and its contribution to the total variance is similar to that obtained during the course of treatment. The same configuration of connections in the factor indicates the continuation of positive trends in hemangioma regression under the influence of the treatment. The action of the "weight" factor in this age group leads to a decrease in apoptosis indicators with an increase in the age of the child. The total contribution of factors after treatment is also $71 \%$.

In the first and second age groups, the greatest contribution to the total variance was made by the "age" factor, which is considered to be the main one. In the third age group (Fig. 3,c) during the course of treatment, the greatest contribution $(41 \%)$ to the total variance is made by the first factor - the "hemangioma severity factor", the influence of which leads to a decrease in the cosmetic effect of hemangioma treatment against the background of its high severity and an increase in the indicator of apoptosis inhibition of sFas. This coincides with modern ideas about the need for early treatment of hemangioma and a decrease in the cosmetic effect of treatment with its late initiation $[37,38]$. The influence of the "age" factor is manifested by a decrease in the activity of hemangioma with an increase in the age of the child. The contribution of both factors to the total variance is $70 \%$. After treatment in the older age group, in comparison with the state during the treatment and with other groups, there is an increase in the number of connections in the structure from 5 to 6 , which indicates an increase in the determinism of the system. The influence of the "treatment efficacy" factor leads to a decrease in its cosmetic effect and an increase in the severity of the manifestations of hemangioma against the background of blocking the manifestations of apoptosis. The structure of connections in the second factor confirms the idea of a decrease in hemangioma activity with an increase in the age of the child. The total contribution of both factors to the variance is $83 \%$, which indicates an insignificant influence of the random component.

Thus, the analysis of the structure of relationships between indicators in factors obtained in different age groups during and after treatment confirms modern clinical concepts of the course and outcomes of treatment of hemangioma in children.

\section{Conclusions}

1. Before treatment, the activity of hemangioma decreases with the age and, accordingly, the severity of hemangioma decreases.

2. The activity and severity of hemangioma decreases after treatment, but with predominance in the younger age group (up to 1 year), which affects the outcome of treatment, that is, when starting treatment of hemangioma in the early stages, the cosmetic effect increases significantly.

3. A decrease in sFasL in the younger age group may indicate a decrease or absence of apoptosis processes, which is clinically expressed by the opposite manifestations - active proliferation. An increase in sFas in the older age group (over a year old) may indicate a blockage of apoptosis processes and, as a consequence, a slow regression of hemangioma.

\section{Conflict of interests}

The authors of the article declare no conflict of interests. 


\section{References}

1. Hinen, H. B., Trenor III, C. C., \& Lee, L. W. (2020). Childhood Vascular Tumors. Frontiers in Pediatrics, 8.

2. Davila-Osorio, V. L., Iznardo, H., Roe, E., Puig, L., \& Baselga, E. (2020). Propranololresistant infantile hemangioma successfully treated with sirolimus. Pediatric Dermatology.

3. Blei, F. (2020). Nomenclature of Vascular Anomalies: Evolution to the ISSVA 2018 Classification System. In Vascular Anomalies (pp. 1-8). Springer, Cham.

4. Lee, M. T., \& Maguiness, S. (2020). Infantile and Congenital Hemangiomas: Natural History, Complications, and When and How to Treat. In Vascular Anomalies (pp. 41-61). Springer, Cham.

5. Wadhwani, M., \& Singh, R. (2020). Capillary hemangioma-A review. CLEVER Clinical and Experimental Vision and Eye Research, 3(1), 13.

6. Adams, D. M., \& Ricci, K. W. (2018). Infantile hemangiomas in the head and neck region. Otolaryngologic Clinics of North America, 51(1), 77-87.

7. Atas, E., Koc, O., \& Artik, H. A. (2017). Clinical features and treatment results in children with hemangioma. Turkish Journal of Pediatrics, 59(3).

8. Alsuwailem, A., Myer $3^{\text {rd }}$, C. M., \& Chaudry, G. (2020, September). Vascular anomalies of the head and neck. In Seminars in Pediatric Surgery (p. 150968). WB Saunders

9. Grzesik, P., \& Wu, J. K. (2017). Current perspectives on the optimal management of infantile hemangioma. Pediatric Health, Medicine and Therapeutics, 8, 107.

10. Chamli, A., Aggarwal, P., Jamil, R. T., \& Litaiem, N. (2019). Hemangioma.

11. Grzesik, P., \& Wu, J. K. (2017). Current perspectives on the optimal management of infantile hemangioma. Pediatric health, medicine and therapeutics, 8, 107-116. https://doi.org/10.2147/ PHMT.S115528

12. Strumila, A., Kazlauskas, V., Posiunas, G., Verkauskas, G., \& Beisa, V. (2017). Infantile hemangioma: Predicting proliferation by infrared thermography. Medicina, 53(2), 85-89.

13. Rosenblatt, A., Mathes, E. F., \& Rosbe, K. W. (2012). Infantile hemangiomas: from pathogenesis to clinical features. Research and Reports in Neonatology, 2, 55.

14. Padhiyar, J. K., Patel, N. H., Gajjar, T. P., Buch, M. D., Shah, Y. B., \& Solanki, R. (2018). Efficacy and safety of propranolol on the proliferative phase of infantile hemangioma: a hospital-based prospective study. Indian Journal of Paediatric Dermatology, 19(3), 224.

15. O'Brien, K. F., Shah, S. D., Pope, E., Phillips, R. J., Blei, F., Baselga, E., ... \& Treat, J. R. (2019). Late growth of infantile hemangiomas in children $>3$ years of age: A retrospective study. Journal of the American Academy of Dermatology, 80(2), 493-499.

16. Wildgruber, M., Sadick, M., Muller-Wille, R., \& Wohlgemuth, W. A. (2019). Vascular tumors in infants and adolescents. Insights into imaging, 10(1), 30.

17. Krowchuk, D. P., Frieden, I. J., Mancini, A. J., Darrow, D. H., Blei, F., Greene, A. K., ... \& Pate, B. M. (2019). Clinical practice guideline for the management of infantile hemangiomas. Pediatrics, 143(1).

18. Fei, Q., Lin, Y., \& Chen, X. (2020). Treatments for infantile Hemangioma: A systematic review and network meta-analysis. EClinicalMedicine, 26, 100506.

19. Wang, C., Li, Y., Xiang, B., Xiong, F., Li, K., Yang, K., ... \& Ji, Y. (2017). Quality of life in children with infantile hemangioma: a case control study. Health and quality of life outcomes, 15(1), 221.

20. Chang, L. C., Haggstrom, A. N., Drolet, B. A., Baselga, E., Chamlin, S. L., Garzon, M. C., ... \& Nopper, A. J. (2008). Growth characteristics of infantile hemangiomas: implications for management. Pediatrics, 122(2), 360-367.

21. Tollefson, M. M., \& Frieden, I. J. (2012). Early growth of infantile hemangiomas: what parents' photographs tell us. Pediatrics, 130(2), e314-e320.

22. Mull, J. L., Chamlin, S. L., Lai, J. S., Beaumont, J. L., Cella, D., Rancour, E. A., ... \& Haggstrom, A. N. (2017). Utility of the Hemangioma Severity Scale as a triage tool and predictor of need for treatment. Pediatric dermatology, 34(1), 78-83.

23. Wu, H. W., Liu, C., Wang, X., Zhang, L., Yuan, W., Zheng, J. W., ... \& Fan, X. D. (2017). Topical application of $0.5 \%$ timolol maleate hydrogel for the treatment of superficial infantile hemangioma. Frontiers in Oncology, 7, 137.

24. Yildirimcakar, D., Demirsoy, U., Azizoglu, M., \& Corapcioglu, F. (2020). Evaluation of Clinical Properties and Treatment Responses of Infantile Hemangioma. Journal of Drugs in Dermatology: JDD, 19(12), 1156-1165. 
25. Zhao, J., Huang, A. H., Rainer, B. M., Kryatova, M. S., Eghrari, A. O., Wang, J., ... \& Cohen, B. A. (2019). Periocular infantile hemangiomas: Characteristics, ocular sequelae, and outcomes. Pediatric Dermatology, 36(6), 830-834.

26. Al-Mayoof, A. F., Joda, A. E., \& Almushhadany, O. E. (2019). Propranolol therapy in infantile hemangioma: correlation of age and duration of treatment to the outcomes. Annals of Pediatric Surgery, 15(1), 1-9.

27. Chang, L., Lv, D., Yu, Z., Ma, G., Ying, H., Qiu, Y., ... \& Lin, X. (2018). Infantile hemangioma: factors causing recurrence after propranolol treatment. Pediatric research, 83(1), 175-182.

28. Park, M., Jung, H. L., Shim, Y. J., Kim, H. S., Yoon, H. S., Park, S. K., ... \& Choi, Y. B. (2020). Serum cytokine profiles in infants with infantile hemangiomas on oral propranolol treatment: VEGF and bFGF, potential biomarkers predicting clinical outcomes. Pediatric Research, 1-7.

29. Pavlidis, L., Spyropoulou, G. A., Papas, A., \& Demiri, E. (2018). Urinary Excretion of MicroRNA126 Is a Biomarker for Hemangioma Proliferation. Plastic and Reconstructive Surgery, 141(2), 319e320 e.

30. Ji, Y., Chen, S., Wang, Q., Xiang, B., Xu, Z., Zhong, L., ... \& Qiu, L. (2018). Intolerable side effects during propranolol therapy for infantile hemangioma: frequency, risk factors and management. Scientific reports, 8(1), 1-7.

31. Schwartz, S. R., Blei, F., Ceisler, E., Steele, M., Furlan, L., \& Kodsi, S. (2006). Risk factors for amblyopia in children with capillary hemangiomas of the eyelids and orbit. Journal of American Association for Pediatric Ophthalmology and Strabismus, 10(3), 262-268.

32. Ding, Y., Zhang, J. Z., Yu, S. R., Xiang, F., \& Kang, X. J. (2019). Risk factors for infantile hemangioma: a meta-analysis.

33. Krowchuk, D. P., Frieden, I. J., Mancini, A. J., Darrow, D. H., Blei, F., Greene, A. K., ... \& Whelan, M.A. (2019). Clinical practice guideline for the management of infantile hemangiomas. Pediatrics, 143(1).

34. Expression and significance of Bcl-2, Bax, Fas and caspase-3 in different phases of human hemangioma / H. Yang, C. Deng, S. Shen [et al.] // J Huazhong Univ Sci Technolog Med Sc. - 2006. Vol. 26, No. 4. - P. 402-404. doi: 10.1007/s11596-006-0405-y.

35. Soluble Fas/FasL ratio as a marker of vasculopathy in children and adolescents with sickle cell disease / A. A. Adly, E. A. Ismail, N. G. Andrawes [et al.] // Cytokine. - 2016. - No. 79. - P. 52-58. doi: 10.1016/j.cyto.2015.12.022.

36. Liao H. FasL/Fas pathway is involved in dengue virus induced apoptosis of the vascular endothelial cells / H. Liao, J. Xu, J. Huang // J Med Virol. - 2010. - Vol. 82, No. 8. - P. 1392-1399. doi: 10.1002/ jmv.21815.

37. Tiemann, L., \& Hein, S. (2020). Infantile Hemangioma: A Review of Current Pharmacotherapy Treatment and Practice Pearls. The Journal of Pediatric Pharmacology and Therapeutics, 25(7), 586-599.

38. Leaute-Labreze, C., Torres, E. B., Weibel, L., Boon, L. M., El Hachem, M., van der Vleuten, C., ... \& Rubin, A. T. (2020). The infantile hemangioma referral score: a validated tool for physicians. Pediatrics, 145(4).

Received: 08-Jan-2021

Accepted: 13-Mar-2021 